What's the Gist? The influence of schemas on the neural correlates underlying true and false memories

Christina E. Webb, Indira C. Turney, \& Nancy A. Dennis

The Department of Psychology, The Pennsylvania State University

University Park, PA

Corresponding author:

Nancy A. Dennis, PhD

Associate Professor

Department of Psychology

The Pennsylvania State University

450 Moore Bldg.

University Park, PA 16802

(office) 814.865 .1712

(fax) 814.863 .7002

nad12@psu.edu 


\section{Abstract}

The current study used a novel scene paradigm to investigate the role of encoding schemas on memory. Specifically, the study examined the influence of a strong encoding schema on retrieval of both schematic and non-schematic information, as well as false memories for information associated with the schema. Additionally, the separate roles of recollection and familiarity in both veridical and false memory retrieval were examined. The study identified several novel results. First, while many common neural regions mediated both schematic and non-schematic retrieval success, schematic recollection exhibited greater activation in visual cortex and hippocampus, regions commonly shown to mediate detailed retrieval. More effortful cognitive control regions in the prefrontal and parietal cortices, on the other hand, supported non-schematic recollection, while lateral temporal cortices supported familiarity-based retrieval of non-schematic items. Second, both true and false recollection, as well as familiarity, were mediated by activity in left middle temporal gyrus, a region associated with semantic processing and retrieval of schematic gist. Moreover, activity in this region was greater for both false recollection and false familiarity, suggesting a greater reliance on lateral temporal cortices for retrieval of illusory memories, irrespective of memory strength. Consistent with previous false memory studies, visual cortex showed increased activity for true compared to false recollection, suggesting that visual cortices are critical for distinguishing between previously viewed targets and related lures at retrieval. Additionally, the absence of common visual activity between true and false retrieval suggests that, unlike previous studies utilizing visual stimuli, when false memories are predicated on schematic gist and not perceptual overlap, there is little reliance on visual processes during false memory retrieval. Finally, the medial temporal lobe exhibited an interesting dissociation, showing greater activity for true compared to false recollection, as well as for false compared to true familiarity. These results provided an indication as to how different types of items are retrieved when studied within a highly schematic context. Results both replicate and extend previous true and false memory findings, supporting the Fuzzy Trace Theory.

Keywords: schema; false memory; fMRI; recollection; familiarity 


\section{Introduction}

Schemas are useful memory tools that allow information to be categorized according to a common concept or theme. In fact, schematic gist or the fundamental content about a scene can be extracted in less than 100 ms (Loftus \& Mackworth, 1978; Potter, 1976; Torralba, Oliva, Castelhano, \& Henderson, 2006). With respect to memory, schematic gist has been shown to guide attention during scene processing and to facilitate memory for schema-consistent information versus schema-unrelated information (Brewer \& Treyens, 1981; Hess \& Slaughter, 1990; Miller \& Gazzaniga, 1998). Despite this processing benefit, similar research has shown that schemas also contribute to false memories as individuals incorrectly identify new, but related, information as 'old'. This research has found high rates of false alarms for lures that include schema-consistent information (Lampinen, Copeland, \& Neuschatz, 2001; Miller \& Gazzaniga, 1998; Neuschatz, Lampinen, Preston, Hawkins, \& Toglia, 2002; Pezdek, Whetstone, Reynolds, Askari, \& Dougherty, 1989), suggesting that schemas may also have a negative influence on verbatim memory. While behavioral and neuroimaging studies have investigated the influence of both semantic and perceptual gist on accurate and illusory memories (for review see Dennis, Bowman, \& Turney, 2015; for meta-analysis see Kurkela \& Dennis, 2016), it is unclear the extent to which highly schematic scenes elicit similar gist and how this gist supports schema memory, distinct from non-schema memory, nor is it clear the role that schemas play in supporting false distinct from true schematic memory. In addition, different memory processes, such as recollection and familiarity, have been shown to distinctly influence memory retrieval (see Yonelinas, 2002 for review). Thus, an understanding of schema's influence on memory should also take into account both recollection and familiarity. To investigate these questions, the current study aimed to: 1) identify the neural correlates of successful memory retrieval of both schematic and non-schematic items when studied within a highly schematic context, 2) investigate differences in retrieval processes between veridical and illusory memory stemming from schematic encoding, and 3) within each type of retrieval, examine the separate roles of recollection and familiarity. To do this, we utilized a novel scene paradigm extended from work from 
Brewer and Treyens (1981) and Miller and Gazzaniga (1998) in which memory was tested for targets and lures that are conceptually related or unrelated to a schematic scene.

Previous behavioral research has noted the benefits of schemas in facilitating both scene and object recognition. For example, Oliva and Torralba (2001) suggested that the global meaning (i.e., gist) of a scene can be extracted even in the absence of object shape or identity. It has also been shown that memory performance is influenced by the conceptual gist of a scene, leading to schema based expectations (Bower, Black, \& Turner, 1979; Brewer \& Treyens, 1981; Hannigan \& Reinitz, 2003; Lampinen et al., 2001; Lampinen, Faries, Neuschatz, \& Toglia, 2000; Miller \& Gazzaniga, 1998; Neuschatz et al., 2002; Pezdek et al., 1989). For example, in one of the original studies investigating the influence of scene gist on memory, Brewer and Treyens (1981) examined participants' memory for items within a schematic context (a graduate student office). Specifically, encoding items included those that were consistent with the schema (e.g., typewriter; hereafter referred to as schematic items), as well as items that would be unusual to find in that context (e.g., picnic basket; hereafter referred to as schemainconsistent items) and items that were not specific to the schema, yet were not out of place or unusual (e.g., rug; hereafter referred to as non-schematic items). Researchers found that participants remembered more schematic items than schema-inconsistent or non-schematic items. Moreover, schema expectancy also contributed to false memories, wherein participants falsely remembered more non-presented schematic objects (e.g., books) than schema-inconsistent or non-schematic objects. Later studies have replicated and extended these initial findings, showing that the rate of false alarms is near equal to the hit rate for schematic objects (Lampinen et al., 2001; Miller \& Gazzaniga, 1998; Pezdek et al., 1989).

While much of the previous research has compared schematic retrieval to retrieval of schemainconsistent information (e.g., octopus on a farm; Brewer \& Treyens, 1981; Loftus \& Mackworth, 1978b; Miller \& Gazzaniga, 1998; Palmer, 1975), it is typically unrealistic to encounter anomalies such as these in everyday life. Rather, one is likely to encounter non-schematic items embedded within schematic scenes, such as a rug in the kitchen or a flowerpot on a farm. Neuroimaging research examining such comparisons is less common in the literature. Those that have find that retrieval of strongly-related (i.e., 
schematic) objects compared to weakly-related (i.e., non-schematic) objects is supported by activity in parahippocampal and retrosplenial complex (Bar \& Aminoff, 2003; Bar, Aminoff, \& Schacter, 2008), reflecting processing and evaluation of associations. In addition, results from encoding suggest a role of the medial PFC in reflecting the strength of relatedness between encoded items (van Kesteren et al., 2013); however results have been mixed at retrieval (e.g., van Buuren et al., 2014; van Kesteren, Rijpkema, Ruiter, \& Fernandez, 2010). With respect to schematic representations, prior work with semantic dementia and language processing has suggested that this strength in semantic relatedness, and in turn semantic and conceptual gist, is associated with activity in lateral temporal cortices, specifically the middle and superior temporal gyri (Noppeney et al., 2007; Price, 2000; see Saumier \& Chertkow, 2002 for review; Simons et al., 2005; Wise \& Price, 2006). Thus, in line with this past work, we expect that retrieval of schematic (compared to non-schematic) true memories should be reliant on associative processing in the medial temporal lobe (MTL) and retrieval of conceptual gist, mediated by the lateral temporal cortices. Additionally, because the role of mPFC in mediating schematic compared to nonschematic retrieval is less clear, any identified results will help further ascertain the function of this region at retrieval.

Compared to schematic true memory, successful memory for non-schematic items, which are only loosely associated with the schema, may not be afforded this same schematic processing advantage, as suggested by reduced retrieval rates observed in prior studies (see Alba \& Hasher, 1983 for review; e.g., Brewer \& Treyens, 1981). Previous research has suggested that reduced memory for non-schematic items reflects both a deficiency in schema support and reduced attention to non-schematic items during encoding (Brewer \& Treyens, 1981; Loftus \& Mackworth, 1978; Owens, Bower, \& Black, 1979; but see Pezdek et al., 1989 in relation to attention for schema-inconsistent items). As a result of reduced attention and weaker encoding, non-schematic retrieval is likely to be associated with more effortful retrieval and monitoring, mediated by activity in the anterior cingulate and parietal cortices (Cabeza, Ciaramelli, Olson, \& Moscovitch, 2008; Ciaramelli, Grady, \& Moscovitch, 2008; Fleck, Daselaar, Dobbins, \& Cabeza, 2006; Wheeler \& Buckner, 2004). Furthermore, due to the small number of studies that have 
compared schematic to non-schematic memory, it was unclear whether the foregoing predictions would be observed in both recollection- and familiarity-based retrieval, or whether memory strength would have differential influences on the neural differences between trial types. Thus, it is important to explore both commonalities and differences in neural recruitment during successful retrieval of schematic and nonschematic items within the context of both recollection and familiarity.

As noted above, while schema processing and schematic gist may serve as useful mechanisms for supporting true memories, they have also been shown to lead to high rates of false memories for schematically-related lures (Aminoff, Schacter, \& Bar, 2008; Intraub, Gottesman, Willey, \& Zuk, 1996; Lampinen et al., 2001; Miller \& Gazzaniga, 1998; Neuschatz et al., 2002; Roediger \& Mcdermott, 1995a). Though examined behaviorally using scenes (e.g., Brewer \& Treyens, 1981; Miller \& Gazzaniga, 1998), it is unclear what specific cognitive and neural mechanisms support the retrieval of schematic false memories. Previous studies examining the neural basis of false retrieval have focused on either semantic false memories or perceptual false memories. Such studies have found substantial overlap in the neural mechanism supporting both true and false memories, including activity throughout the core retrieval network, including bilateral frontal and parietal regions (see Dennis et al., 2015; Kurkela \& Dennis, 2016 for reviews), bilateral caudate and insula (e.g., Cabeza, Rao, Wagner, Mayer, \& Schacter, 2001; GaroffEaton, Slotnick, \& Schacter, 2006; Slotnick \& Schacter, 2004), lateral temporal cortex (e.g., von Zerssen, Mecklinger, Opitz, \& von Cramon, 2001), occipital cortex (Cabeza et al., 2001; Dennis, Bowman, \& Peterson, 2014; Garoff-Eaton et al., 2006; Schacter et al., 1996; Stark, Okado, \& Loftus, 2010) and hippocampus/parahippocampal gyrus (PHG) (Dennis, Bowman, \& Vandekar, 2012; Garoff-Eaton et al., 2006; Iidaka, Harada, Kawaguchi, \& Sadato, 2012; Schacter, Buckner, Koutstaal, Dale, \& Rosen, 1997; Slotnick \& Schacter, 2004; Stark et al., 2010; von Zerssen et al., 2001). While much of this activation has been attributed to engagement of similar reconstructive processes (Cabeza et al., 2001; Dennis et al., 2012; Garoff-Eaton et al., 2006; Gutchess \& Schacter, 2012; Kahn, Davachi, \& Wagner, 2004; Schacter et al., 1997; Schacter et al., 1996; Slotnick \& Schacter, 2004; von Zerssen et al., 2001), as well as 
retrieval-related evaluation and monitoring processes (Garoff-Eaton et al., 2006; Stark et al., 2010), basic stimulus-driven processes are also believed to account for the large amount of common neural activity.

For example, in perceptual relatedness paradigms, targets and lures share similar perceptual attributes, such as shape, color and/or size. This, in turn, is believed to result in similar perceptual processing in late visual cortices (BA $19 \& 37$ ) between targets and related lures (e.g., Atkins \& ReuterLorenz, 2011), reflecting the conscious experience of memory, which is independent of true "oldness" (Dennis et al., 2012; Slotnick \& Schacter, 2004). It is also thought to be indicative of successful retrieval of the general properties of originally studied items (e.g., shape, color; Slotnick \& Schacter, 2004) or those involving the semantic label or general category (e.g., fruit, bird) to which the item belongs. However, what is unique about the scene paradigm is that, unlike other visual false memory studies, the critical lures are not items perceptually related to that which was presented previously, but rather conceptually related to encoding items. For example, 'tractor' is schematically related to 'farm' and objects within a farm scene such as 'barn' and 'pig', but shares little perceptual similarity with these objects.

Therefore, it follows that schematic false memories, like those in the scene paradigm, arise not from activity in visual processing regions associated with perceptual overlap between lures and encoding information (Dennis et al., 2012; Goldmann et al., 2003; Gutchess \& Schacter, 2012; Slotnick \& Schacter, 2004), but from a shared conceptual or semantic gist between the encoding schema and the lures. In line with the influence of such semantic processing on memory retrieval, previous studies have highlighted the role of the lateral temporal cortices in mediating both true (as noted above) and illusory memories. Specifically, engagement of left lateral temporal cortex has been associated with semantic processing and retrieval of semantic gist across both true and false memory studies (Cabeza et al., 2001; Dennis et al., 2012; Dennis, Kim, \& Cabeza, 2008b; Garoff-Eaton, Kensinger, \& Schacter, 2007; Gutchess \& Schacter, 2012; Kim \& Cabeza, 2007b; Kubota et al., 2006). With respect to schematic true and false memory, given the schematic salience invoked in the scene paradigm, we hypothesize that both types of memory will be highly reliant on the retrieval of schematic gist. Importantly, given that schematic gist, as 
operationalized in the current scene paradigm, is inherently built on the semantic associations between items that are congruent with the presented schema at encoding (e.g., 'barn' and 'pig' with respect to 'farm'), we hypothesize that retrieval of both schematic targets and lures will be mediated by activity in bilateral temporal cortices, reflecting gist processing.

As noted above, MTL has also been shown to be a critical region, not just in supporting true, but also false memories (Dennis et al., 2014; Dennis et al., 2008b; Dennis, Kim, \& Cabeza, 2007b; Duarte, Graham, \& Henson, 2010; Garoff-Eaton et al., 2007; Norman \& Schacter, 1997). However, the exact role of the MTL with respect to false memories remains unclear. One issue that may be contributing to this confusion is the fact that different MTL subregions have been shown to contribute differentially to recollection and familiarity in veridical memories (e.g., Daselaar, Fleck, \& Cabeza, 2006; see Yonelinas, 2002 for review), and thus, may also do so with respect to false memories. That is, while a wide breadth of behavioral research has shown that, like true memories, false memories can be retrieved in terms of both (phantom) recollection and familiarity (Brainerd, Wright, Reyna, \& Mojardin, 2001; Lampinen et al., 2001; Mather, Henkel, \& Johnson, 1997; see also McCabe, Roediger, McDaniel, \& Balota, 2009) neuroimaging studies have just begun to investigate this dichotomy (Abe et al., 2013; Dennis et al., 2012; Duarte et al., 2010; Garoff-Eaton et al., 2007; Kim \& Cabeza, 2007b; Stark et al., 2010). For example, Kim and Cabeza (2007b) compared high-confidence true recognition with high-confidence false recognition and found that true memory elicited increased activation in bilateral posterior hippocampus and PHG, indicating that high-confidence true recognition is mediated by recollection-related processes, whereas phantom recollection is not. Alternatively, recent work from our lab (Dennis et al., 2012) has shown that, compared to familiarity, both true and false recollection exhibited activity in the anterior hippocampus and PHG; yet true recollection showed greater hippocampal activity indicative of greater recovery of sensory details associated with past events.

Studies that have identified differences in familiarity-based true and false memories have told a slightly different story with respect to MTL activity. Specifically, recent work has suggested that false familiarity elicits greater activation in PHG due to a reliance on source retrieval in light of weak encoding 
of the item (Abe et al., 2013; Karanian \& Slotnick, 2014; see also Kim \& Cabeza, 2007b). Given the role of the PHG in source retrieval (Diana, Yonelinas, \& Ranganath, 2007; Eichenbaum, Sauvage, Fortin, Komorowski, \& Lipton, 2012; Ranganath, 2010; Yonelinas, Hopfinger, Buonocore, Kroll, \& Baynes, 2001), it has been posited that PHG activity reflects effortful contextual processing associated with attempts to relate the lure with an encoding context (Karanian \& Slotnick, 2014). The schematic encoding component of the current design allows for the examination of this theory of MTL activity and false memories. While a reliance on schematic gist of past presentations can easily lead to phantom recollection of schematic lures, it is also likely to result in high rates of familiarity-based false memories, as the strong schematic gist guides scene reinstatement and retrieval processes. To the extent that the encoding scenes elicit schematic contextual support during retrieval, we hypothesize that the hippocampus will show greater activation for true compared to false recollection and false familiarity will exhibit a greater reliance on the PHG than true familiarity.

To recap, the current study aims to expand upon previous false memory studies by investigating the influence of a schematic framework on memory retrieval utilizing a scene paradigm within the context of fMRI. To this end, we will extend the research on veridical memories to include an investigation of the neural basis of common and distinct mechanisms supporting schematic and non-schematic memories within the context of both recollection and familiarity. We will also extend the research on false memories to include an examination of the neural basis of visual false memories beyond previous work focusing on the role of perceptual relatedness. In addition to the foregoing investigation into veridical memories, we also aim to investigate both common and unique factors underlying true and false memories within the context of a schematic framework across both recollection and familiarity.

\section{Materials and Methods}

2.1. Participants. Twenty-five right-handed native English speakers from the Penn State University community completed this experiment. Participants were screened for history of neurological disorders and psychiatric illness, alcoholism, drug abuse, and/or learning disabilities. One participant was excluded 
from the analysis due to head motion in excess of $4 \mathrm{~mm}$. Two additional participants were also excluded for poor behavioral performance (greater than 50\% miss rate for schematic targets), leaving data from 22 participants reported in all analyses [13 females; mean age $=22.91$ years $(\mathrm{SD}=3.01)]$. All participants provided written informed consent and received financial compensation for their participation. All experimental procedures were approved by The Pennsylvania State University's Institutional Review Board for the ethical treatment of human participants.

2.2. Stimuli. Stimuli consisted of 26 schematic scenes (e.g., Bathroom, Farm), comprised of objects commonly associated with each schema (schematic targets: e.g., Farm: pig, barn; Bathroom: toilet, tub) as well as items unrelated to the schema (non-schematic targets: e.g., Farm: bush; flower pot; Bathroom: vase; spray bottle) (see Figure 1). Lures also consisted of both items commonly associated with each schema (schematic lures: e.g., Farm: rooster, tractor; Bathroom: sink, plunger), as well as unrelated lures (e.g., purse, hammer). All backgrounds and images were obtained from an Internet image search. All items included in testing were normed for their association with each scene in a multi-step process. Specifically, all schematic scenes, along with their related schematic items, were verbally identified by both a group of individuals in the lab as well as an independent group of subject pool participants $(\mathrm{N}=$ 20) in order to verify that each scene represented the intended schema. Furthermore, schematic items were identified as being related to the schema via a simple yes/no identification procedure. Items that were deemed as not related to the schema (i.e., that did not reach $100 \%$ consensus) were replaced with an appropriate alternative.

Once the schematic targets and lures were finalized, non-schematic targets were then inserted in each scene. Non-schematic targets were defined as those that did not contribute to the schema of either the scene they were inserted into or any other scene in the study, yet still fit appropriately into the scene (i.e., such that at retrieval they did not stand out as schematic or schema-inconsistent to any given scene). Due to the difficulty of determining these non-schematic items, we transitioned to a rating scale. Specifically, non-schematic items were rated on an ordinal scale (1-unrelated to 4- related) by a unique 
group of subject pool participants $(\mathrm{N}=19)$ to ensure that non-schematic items were not schematically related to the scene in which they appeared, or any other scene. This rating process also included a random subsample of the schematic items, so that participants were not biased in their ratings of nonschematic items. As a first step in creating a division with respect to schema relatedness, only nonschematic items that met a rating of below 2.5 across all scenes were included as non-schematic targets. Items that failed to meet this criterion were replaced with appropriate alternatives. With respect to final relatedness ratings, while the subsample of schematic items had a mean relatedness rating of $3.70(\mathrm{SD}=$ 0.41 ; mode $=3.89)$, non-schematic items had a mean rating of $1.48(\mathrm{SD}=0.32$; mode $=1.14)$. This difference was statistically significant $(\mathrm{t}(269)=38.24 ; \mathrm{p}<.001)$. To further investigate this issue, we examined the average ratings corresponding to each recollection and familiarity hit within both schematic and non-schematic items. Similar to the overall schema-relatedness ratings mentioned above, significant differences in relatedness were identified between schematic \& non-schematic recollection, as well as familiarity, hits. A list of all items and scenes can be found in Appendix A. Scenes were presented focally and equated for resolution. Twenty-six scenes were presented during encoding in the same order for all participants. Three hundred individual items were presented at retrieval, including (a) 104 related targets (4 from each scene); (b) 62 unrelated targets (2-3 from each scene); (c) 104 related lures (4 associated with each scene); and (d) 30 unrelated lures.

\subsection{Task and Procedure. Encoding took place outside of the scanner while retrieval occurred in the} scanner with approximately 30 minutes separating the two memory phases. Encoding was intentional; participants were asked to look at each scene and try to remember as much as they could for a later memory task. The 26 encoding scenes were presented for $10 \mathrm{~s}$ each across 2 runs, with 13 scenes presented in run one and the remaining 13 scenes presented in run two. During retrieval, all images (items from the scenes and new items) were presented in the center of the screen with three response options (Remember/Know/New) displayed below each image. Images were projected onto a screen that participants viewed through a mirror attached to the head coil. Behavioral responses were recorded with 
the participant's right hand on a 4-button response box. Images were displayed by COGENT in MATLAB (Mathworks Inc., Natick, MA, USA) and were displayed at a screen resolution of $1024(\mathrm{H}) \mathrm{x}$ $768(\mathrm{~V})$ at $75 \mathrm{~Hz}$. At the viewing distance of $143 \mathrm{~cm}$, the display area was $20^{\circ}(\mathrm{H}) \times 16^{\circ}(\mathrm{V})$. Scanner noise was reduced with headphones, and cushioning was used in the head coil to minimize head motion.

During retrieval, participants completed 6 runs each approximately 7 minutes in length. Each image was displayed for $3 \mathrm{~s}$ while participants made memory responses using the 'Remember/Know/New' paradigm. The images were pseudorandomly sorted, ensuring that no more than 3 images from any one trial type appeared in a row and no 2 images associated with a given scene appeared in a row. In accord with typical Remember/Know/New task instructions, participants were told to respond 'Remember' if they could recollect specific details about the object such as its shape, color, placement in the scene or their thoughts or feelings during its initial presentation. Participants were told to respond 'Know' if the picture looked familiar, but they could not recollect any specific details of its prior presentation. They were told to respond 'New' if they believed the picture was not presented during the encoding session.

2.4. Image acquisition. Structural and functional images were acquired using a Siemens $3 \mathrm{~T}$ scanner equipped with a 12-channel head coil. A T1-weighted sagittal localizer was acquired to locate the anterior (AC) and posterior (PC) commissures. Images were then prescribed parallel to the AC-PC plane. An MPRAGE was acquired with a $1650 \mathrm{~ms}$ TR, $2.03 \mathrm{~ms}$ TE, $256 \mathrm{~mm}$ field of view (FOV), $256^{2}$ matrix, 160 axial slices, and $1.0 \mathrm{~mm}$ slice thickness for each participant. Echo-planar functional images were acquired using a descending acquisition, $2500 \mathrm{~ms}$ TR, $25 \mathrm{~ms}$ TE, $240 \mathrm{~mm}$ FOV, a $80^{2}$ matrix, $90^{\circ}$ flip angle, 42 axial slices with $3.0 \mathrm{~mm}$ slice thickness resulting in $3.0 \mathrm{~mm}$ isotropic voxels.

\subsection{Image processing. Pre-processing of all functional images was carried out in SPM8 (Wellcome} Institute of Cognitive Neurology, London, UK. www.fil.ion.ucl.ac.uk), using MATLAB (Mathworks Inc., Natick, MA, USA). The functional time series were first corrected for differences in slice timing acquisition. EPI images were then realigned to the first image using a 6-parameter rigid body affine 
transformation. Next, the slice time corrected functional images were spatially normalized to the standard MNI (Montreal Neurological Institute) EPI template implemented in SPM8. To do this, the raw T1 MPRAGE images were co-registered to the mean realigned functional image, and then this co-registered T1MPRAGE image was segmented and registered to the MNI template. Lastly, the parameters from this registration process were applied to the slice time corrected and realigned functional images $(3 \mathrm{~mm}$ isotropic voxels) to normalize them to the MNI template, with coordinates later converted into Talairach space (Talairach \& Tournoux, 1988) for reporting. As a final preprocessing step, all of the normalized functional images were smoothed using a 6mm full-width-half-maximum Gaussian smoothing kernel.

2.6. fMRI analyses. At the first level, trial-related activity was modeled using the general linear model (GLM) with a stick function corresponding to trial onset convolved with a canonical hemodynamic response function. A second-level random effects GLM was created treating subjects as a random effect. One sample t-tests were conducted to investigate contrasts of interest. The current analyses focused on 8 trial types of interest: 1) Schematic Recollection Hits, which were defined as 'Remember' responses to related targets; 2) Schematic Familiarity Hits, which were defined as 'Know' responses to related targets; 3) Schematic Misses, which were defined as 'New' responses to related targets; 4) Schematic Recollection False Alarms (FA), which were defined as 'Remember' responses to related lures; 5) Schematic Familiarity False Alarms, which were defined as 'Know' responses to related lures; 6) Schematic Correct Rejections (CR), which were defined as 'New' responses to related lures; 7) Nonschematic Recollection Hits, which were defined as 'Remember' responses to unrelated targets; 8) Nonschematic Familiarity Hits, which were defined as 'Know' responses to unrelated targets; and 9) Nonschematic Misses, which were defined as 'New' responses to unrelated targets. All other trial types, along with no response trials, were coded with their own regressors (as were movement parameters) and treated as regressors of no interest.

The first goal of the paper was to elucidate the neural mechanisms supporting memory for schematic and non-schematic items within both recollection and familiarity. To examine neural regions 
that were common to both schematic and non-schematic retrieval success we conducted a conjunction of schematic recollection hits $>$ misses and non-schematic recollection hits $>$ misses. The same analysis was subsequently conducted using familiarity hits. We then directly compared schematic recollection hits with non-schematic recollection hits, as well as schematic familiarity hits with non-schematic familiarity hits using whole-brain maps to identify regions where successful retrieval processes differed between the two trial types. Unfortunately, a majority of participants did not make enough non-schematic false alarms within recollection or familiarity $(<10$ trials) to allow for the investigation of non-schematic false memories. We therefore focused our false memory analyses on only schematic items.

Second, we aimed to elucidate the neural mechanisms supporting schematic false memories. In order to identify neural correlates supporting false recollection, we compared schematic recollection false alarms to schematic correct rejections. Next, a conjunction analysis of true and false recollection (Hit $>$ Miss $\cap$ FA $>$ CR) activity was constructed in SPM in order to identify neural activity that was common to both memory processes. In order to identify neural resources that were differentially recruited for each type of recollection response, we directly compared activity supporting recollected hits and recollected false alarms. To examine true and false familiarity, a similar conjunction analysis was conducted using schematic familiarity hits and schematic familiarity false alarms (in comparison to misses and correct rejections). We then again directly compared activity, this time supporting familiarity hits and familiarity false alarms.

In order to identify significant results in our contrasts of interest, we employed Monte Carlo simulations, implemented by 3dClustSim in AFNI version 16.0 (Cox \& Hyde, 1997) to determine activation that was corrected for multiple comparisons at $p<0.05$. The input to this simulation was the search space [grey matter mask using the Wake Forest University aal pickatlas (Lancaster et al., 2000; Maldjian, Laurienti, Kraft, \& Burdette, 2003)], across-subject average intrinsic smoothness in mm obtained from the residual time-series (11.15), and the uncorrected $p$ threshold $(p<0.005)$. The results revealed a voxel extent of 66 required to correct for multiple comparisons at $p<0.05$ at the whole brain level. An additional simulation was run to determine a correction specific to our a priori ROIs of interest, 
including the superior/middle temporal gyri (STG/MTG) and the MTL (bilateral hippocampus and parahippocampal gyrus). All ROIs were defined by using anatomical masks of each region (bilaterally) within the aal pickatlas toolbox in SPM8.

\section{Results}

3.1. Behavioral Results. A $2 \times 2$ repeated measures ANOVA was conducted across all memory responses (combining recollection and familiarity) to examine the effects of stimulus type (target, lure) and relatedness (schematic, non-schematic) on proportion of 'old' responses (see Table 1 for means). This analysis revealed a significant main effect of stimulus type $[F(1,21)=155.08, p<.001]$, with participants making more 'old' responses to targets than to lures. There was also a significant main effect of relatedness, such that participants made more 'old' responses to schematic items than non-schematic items $[F(1,21)=82.74, p<.001]$, as well as a significant stimulus type by relatedness interaction $[F(1$, $21)=24.35, p<.001]$. Follow up t-tests indicated that participants made more hits than false alarms for both schematic $[t(21)=6.49, p<.001]$ and non-schematic $[t(21)=13.11, p<.001]$ items. Participants also made more hits and false alarms to schematic targets than non-schematic targets $[t(21)=5.58, p<$ $.001 ; t(21)=9.8, p<.001 ;$ respectively $]$

A similar 2 x 2 ANOVA on recollection responses revealed a significant main effect of stimulus type $[F(1,21)=92.39, p<.001]$ and relatedness $[F(1,21)=120.9, p<.001]$. Follow up t-tests indicated that participants made more recollection hits than recollection false alarms for both schematic and nonschematic items $[t(21)=8.45, p<.001 ; t(21)=9.3, p<.001 ;$ respectively $]$ and participants made significantly more schematic hits and schematic false alarms than non-schematic hits and false alarms [Hit: $t(21)=11.69, p<.001$; FA: $t(21)=7.64, p<.001$ ]. Finally, a 2 x 2 ANOVA on adjusted familiarity rates was conducted. Adjusted familiarity hits were calculated as pKnow Hits/(1 - pRemember Hits) and adjusted familiarity FA were calculated as pKnow FA/(1 - pRemember FA). This calculations takes into account the fact the recollection and familiarity are not mutually exclusive processes (Duarte et al., 2010; Duarte, Ranganath, Trujillo, \& Knight, 2006; Yonelinas, 2002; Yonelinas \& Jacoby, 1995). This 
ANOVA revealed a significant main effect of stimulus type $[F(1,21)=47.9, p<.001]$ and relatedness $[F(1,21)=60.6, p<.001]$, as well as a significant interaction $[F(1,21)=26.9, p<.001]$. Follow up ttests showed that adjusted familiarity hit rates were higher than adjusted familiarity false alarm rates in both schematic and non-schematic conditions $[t(21)=2.38, p<.05 ; t(21)=8.14, p<.001$; respectively], though much more so in the non-schematic condition. Results also indicated that participants made more adjusted familiarity hits and false alarms for schematic compared to non-schematic targets and lures $[t(21)$ $=2.38, p<.05 ; t(21)=9.43, p<.001$ respectively]. A breakdown of the behavioral results is shown in Table 1.

\subsection{Imaging Results}

3.2.1. Schematic and Non-Schematic Retrieval Success. With respect to true memories, a conjunction of schematic and non-schematic recollection success (Recollection Hit v Miss) revealed activation in regions within bilateral fronto-parietal cortices, bilateral middle temporal cortices, and left inferior frontal gyrus (IFG). A parallel conjunction examining familiarity success revealed activity in a more limited set of regions, including left middle and inferior frontal gyrus, left superior parietal cortex and left middle temporal gyrus (see Table 2; Figure 2A).

The effect of schema on target recollection revealed greater activation in bilateral occipital cortex and left hippocampus for schematic target recollection, while non-schematic target recollection was supported by dorsal ACC and left supramarginal gyrus ${ }^{1}$. In regards to familiarity, no significant activity was observed for schematic greater than non-schematic target familiarity. The reverse contrast revealed activation only in bilateral middle temporal gyri (see Table 3; Figure 2B).

\footnotetext{
${ }^{1}$ It should be noted that the contrast of non-schematic recollection success (Hit v Miss) did reveal posterior parahippocampal gyrus at the corrected threshold, indicating that non-schematic targets utilized MTL for successful recollection.
} 


\subsubsection{True and False Schematic Memory Retrieval Activity. A conjunction analysis between schematic} true and false recollection revealed activation in the left middle frontal gyrus (MFG), left MTG, and bilateral inferior parietal lobe. Conjunction analyses between schematic true and false familiarity revealed activation in the left MFG and the left MTG (see Table 4; Figure 3A).

Direct comparisons between schematic true and false recollection revealed greater activation in bilateral hippocampus, right MTG, and left occipital gyrus for true recollection, while false recollection was supported by greater activity in bilateral STG. No region showed greater activation for true greater than false familiarity; however false familiarity revealed greater activity in bilateral MTG/STG and left hippocampus, extending into PHG (see Table 5; Figure 3B).

\section{Discussion}

Using a novel scene paradigm, the current study investigated the effect of schematic encoding on memory for schematic and non-schematic information, as well as schematic encoding on schematic false memories. The behavioral analyses showed that when a strong schematic context was present at encoding, memory for schematic items was better than for non-schematic items. While successful retrieval of both types of items was shown to rely on similar neural processes, differences between schematic and nonschematic retrieval revealed that schematic target recollection relied on the hippocampus and visual cortices, with no region showing greater activation for schematic target familiarity. Conversely, nonschematic target recollection was supported by regions in the prefrontal and parietal cortices, whereas non-schematic target familiarity was supported by greater activity in the lateral temporal cortices. While schematic encoding was shown to be beneficial to successful memory, it also significantly increased false memories for schematically-related lures. Neurally, schematic true and false recollection, as well as true and false familiarity, showed overlapping activation, suggesting that both types of memory processes recruit similar neural mechanisms regardless of the process supporting the memory decision. In particular, activity in left MFG and lateral temporal cortices was identified across all four memory responses, highlighting the ubiquitous role of these regions in the retrieval of schematic memories. Additionally, 
analyses identified several brain regions that distinguished between true and false retrieval. Specifically, visual cortex showed increased activity for true recollection, whereas bilateral MTG and STG showed increased activity for false recollection and familiarity. Interestingly, the MTL showed increased activity for true compared to false recollection, but showed the opposite pattern for familiarity. Each finding is discussed in detail below.

\subsection{Common Activity Across Schematic and Non-schematic Retrieval Success. In line with previous} studies that have found that schematic items are often better remembered than non-schematic items (see Alba \& Hasher, 1983 for review; e.g., Brewer \& Treyens, 1981), results showed that schematic targets were remembered more often than non-schematic targets. This difference is reflective of the influence that the encoding schema has in supporting identification of and memory for information that is directly supportive of the schema. Despite this, successful retrieval of both schematic and non-schematic targets was shown to rely on similar neural correlates across both recollection and familiarity. Specifically, retrieval success of both types of targets was mediated by activity in bilateral prefrontal and parietal cortices, including left IFG, regions consistently shown to support episodic recognition processes (Rugg \& Vilberg, 2013; Spaniol et al., 2009).

Activation was also identified in lateral temporal cortices, which have been shown to support semantic processing and retrieval of verbal and semantic gist information (Saumier \& Chertkow, 2002; Wise \& Price, 2006). Moreover, we found a similar activation profile in lateral temporal cortices for both schematic and non-schematic retrieval success across recollection and familiarity. While initially counterintuitive, results suggest that the schematic context instantiated during encoding is a strong mediator of all retrieval processes, regardless of relatedness (schematic/non-schematic), memory strength (recollection/familiarity), and, as will be discussed later, veracity (true/false). Specifically, results suggest that once items are encoded in a highly schematic context, memory for both schematic and non-schematic items from that context is equally reliant on semantic gist processes. Importantly, this finding extends 
previous work by indicating that retrieval of items studied within a highly schematic context utilizes the schema for accurate retrieval support, even when these items are not directly supportive of the schema.

\subsection{Differences in Schematic and Non-schematic Target Retrieval. Behavioral analyses found that} schematic targets were better remembered than non-schematic targets. Furthermore, as predicted, schematic target recollection elicited greater activity in the hippocampus and visual cortex. The MTL, and specifically the hippocampus, has been consistently implicated in recollection success and in the retrieval of item-specific details, as well as source information (Diana et al., 2007; Eldridge, Knowlton, Furmanski, Bookheimer, \& Engel, 2000; Yonelinas, Otten, Shaw, \& Rugg, 2005). Results support the notion that schematic compared to non-schematic hits are associated with the retrieval of greater encoding details, potentially due to enhanced encoding processes associated with identification and processing of the studied schema. In line with previous work, results suggest that schematic target recollection is reliant on greater contextual processing supported by the MTL (Bar \& Aminoff, 2003; Bar et al., 2008).

Schematic target recollection was also supported by increased activity in late visual cortices (BA 19/37), compared with non-schematic target recollection. With respect to memory retrieval, activity in early visual cortex has been interpreted as reflecting recapitulation of item representations that were present at encoding, while activity in late visual cortex has been interpreted as reflecting memory for general object identity and the conscious experience of identifying the item as 'old' (Slotnick \& Schacter, 2004, 2006; Stark et al., 2010; Vaidya, Zhao, Desmond, \& Gabrieli, 2002; Wheeler, Petersen, \& Buckner, 2000). To this end, results suggest that while lower level sensory reactivation of targets was consistent across schematic and non-schematic retrieval success (as evidenced by overlap in activity in early visual cortex), non-schematic target retrieval relied on conscious retrieval of the item and/or its context within the schema to support recollection. Interestingly, the foregoing differences were only observed for recollection and not familiarity responses, suggesting that neural differences in enhanced schema-related item accuracy may be supported by different mechanisms for familiarity. 
Because non-schematic targets were not inherently tied to the encoding schema, it was predicted that participants would not be able to take full advantage of the encoding schema at retrieval, making these items more difficult to remember. This was reflected in both behavior and the neural results. Specifically, poorer recollection memory for non-schematic compared to schematic items was associated with increased activity within the ACC and left supramarginal gyrus. Previous memory research has implicated the ACC in supporting effortful cognitive control and conflict monitoring processes (Carter et al., 1998; Fleck et al., 2006), while the parietal cortex has been interpreted as mediating identification of old, or previously encountered, information. Additionally, the supramarginal gyrus, has been attributed to increases in attentional mechanisms, specifically within the context of recollection (Cabeza et al., 2008; Ciaramelli, Grady, Levine, Ween, \& Moscovitch, 2010; Wheeler \& Buckner, 2004), and may also direct a search for retrieval of general information that shares properties with a variety of contexts and is therefore more difficult to retrieve, as in the case of non-schematic items. Together, these results highlight the more difficult nature, and thus the increased cognitive effort, needed to retrieve non-schematic items when presented within a highly schematic context at encoding.

With respect to familiarity-based retrieval, no regions showed greater activation for schematic than non-schematic familiarity hits. Interestingly, non-schematic items relied on the lateral temporal cortices to a greater degree than did schematic retrieval. While counter-intuitive, we theorize that it may be the case that when presented with a weaker retrieval trace, retrieval of non-schematic items includes search and retrieval of the associated schema in which the items were presented in order to support item retrieval (see Tse et al., 2007; van Kesteren, Ruiter, Fernández, \& Henson, 2012). Future work is needed to examine this possibility with respect to non-schematic retrieval following schema-based encoding.

It should be noted that a handful of previous studies have identified a role for medial prefrontal cortex (mPFC) in supporting schema processing and schema memory retrieval (e.g., van Kesteren et al., 2013; van Kesteren, Rijpkema, et al., 2010). In the current study, however, we did not find evidence of mPFC in mediating retrieval of schematic compared to non-schematic memories (for a similar finding see, van Buuren et al., 2014). While van Kesteren, et al. (2013) also included analogous non-schematic 
items to those used in the current paradigm (identified as schema intermediate items in their study), the observed linear schema-congruency effect in mPFC does not appear to be due to differences in schematic and non-schematic retrieval, but instead appears to be due to a marked deactivation in mPFC associated with schema-incongruent retrieval. This may provide insight into the lack of mPFC observed in the current paradigm and may suggest that $\mathrm{mPFC}$ findings observed in other schema-based memory studies is either related to encoding-retrieval differences or is due to comparisons with schema-inconsistent memories. It is also possible that the $\mathrm{mPFC}$ plays a greater role in facilitation of knowledge integration at encoding and/or consolidation stages (Tse et al., 2011; van Kesteren, Rijpkema, Ruiter, Morris, \& Fernandez, 2014; van Kesteren et al., 2013; van Kesteren, Fernandez, Norris, \& Hermans, 2010) as opposed to retrieval. To continue to clarify these issues, it is important that more studies be conducted to examine schema-relatedness on a continuous scale, as well as identifying neural differences between schema memory encoding and retrieval.

\subsection{Common activity for true and false memory. In addition to its advantages to memory retrieval,} schematic encoding has also been shown to have detrimental effects on memory, particularly with respect to an increased rate of false memories. Accordingly, while the schematic hit rate was greater than that of schematic false alarms, participants made significantly more schematic false alarms than non-schematic false alarms. In line with our predictions, the conjunction analysis of schematic true and false recollection (as well as that of familiarity) showed common retrieval activity in left MFG and left MTG (see Figure 3A). As noted above, common activity in left MFG and left MTG highlights the influence of the encoding schema on memory retrieval, as both regions have been shown to be critical to semantic processing (Binder, Desai, Graves, \& Conant, 2009; Kircher, Brammer, Tous Andreu, Williams, \& McGuire, 2001; Noppeney \& Price, 2002; Price, 2000; Wise \& Price, 2006) and the retrieval of semantic gist (Buckner, 1996; Gabrieli, Poldrack, \& Desmond, 1998; Noppeney et al., 2007; Price, 2000; Simons et al., 2005; Wise \& Price, 2006). Additionally, within the context of false memory studies, the MTG has been shown to mediate retrieval of false memories based on semantic or conceptual similarity between lures and 
targets (Cabeza et al., 2001; Dennis et al., 2008b; Garoff-Eaton et al., 2007; Kim \& Cabeza, 2007b; Kubota et al., 2006). Given that in the scene paradigm schematic gist is inherently built on the semantic associations between items that are congruent with the encoding schema, results suggest that recruitment of semantic processing regions underscores retrieval of the schema and the evaluation and integration of the congruent item (target or lure) to that schema. Moreover, the fact that left MFG and left MTG were identified in both the recollection and familiarity conjunction, speaks to the ubiquitous influence of semantic processing and schematic gist to schematic memory retrieval, in the current paradigm.

Interestingly, the results failed to show common activity in visual cortex, a region that has been identified in several previous false memory studies that have employed visual stimuli (Dennis et al., 2012; Gutchess \& Schacter, 2012; Kurkela \& Dennis, 2016; Slotnick \& Schacter, 2004). As noted in the Introduction, while the scene paradigm is indeed visual by nature, is constitutes a relatively different paradigm than the perceptual relatedness paradigms used to date. A 'visual DRM' if you will, the scene paradigm examines memory for targets and lures that were related to a given schema (e.g., 'toilet' and 'sink' with respect to 'Bathroom') and are associated by means of common conceptual or schematic gist amongst the items. However, the items themselves share little perceptual similarity. Our current results suggest that, in the absence of perceptual overlap between targets and lures, there exists no overlapping processes within visual cortex that support recollection or familiarity of both true and false memories. This finding thus extends results from previous visual false memory studies and deepens our understanding of what processes may be common to false memories across a wide range of paradigms, as well as what processes underlie false retrieval with respect to the specific paradigm used.

4.4. Differential activation between schematic true and false memory. In addition to common activation, we sought to elucidate neural activity that differentially supported schematic true and false memories. Important differences between true and false schematic memory were identified in visual cortex, lateral temporal cortices, and MTL, with each discussed in turn below. Despite the lack of common visual activation, recollection results showed that true compared to false recollection showed increased activity 
across both early and late visual cortex (BA 18 \& 19). As noted above, early visual activity has been attributed to the recapitulation of a sensory signature reflecting the retrieval of sensory details of the encoding event (c.f., the sensory reactivation hypothesis; Buckner \& Wheeler, 2001; Rugg \& Wilding, 2000), while late visual activity has been attributed to the retrieval of item identity (Slotnick \& Schacter, 2004; Vaidya et al., 2002; Wheeler \& Buckner, 2003; Wheeler et al., 2000). To this end, the results suggest that, compared to false recollection, true recollection is mediated by reactivation and reinstatement of perceptual details associated with the original encoding episode, as well as object labeling and general properties of the originally studied item (Garoff-Eaton et al., 2006; Slotnick \& Schacter, 2004). Furthermore, given the fact that visual cortex activity failed to differentiate true and false familiarity, results suggest that familiarity responses may not retain the same perceptual details that are present for recollection ${ }^{2}$. Combined with the fact that no other region distinguished true from false familiarity, the results further suggest that, unlike recollection, subjective differences between familiarity decisions are less distinctive and are largely reliant on similar cognitive and neural processes.

Most interestingly, both recollection and familiarity false memories exhibited greater activity in bilateral lateral temporal cortices compared to true memories. Specifically, schematic false compared to true recollection exhibited increased activation in bilateral STG and schematic false compared to true familiarity exhibited increased activation in both bilateral MTG and STG (see Figure 3B). Thus, results are consistent with our prediction that, given the salient schematic relationship between lures and the studied scenes, false memories would be largely reliant on retrieval of the schematic gist (Deese, 1959; Roediger \& McDermott, 1995b). Additionally, combined with the absence of visual processes supporting false retrieval, results further suggest that false memories for visual stimuli that are schematically, but not overtly perceptually related to information presented at encoding are mediated by the retrieval of schematic gist, regardless of memory strength. As such, the current results are consistent with the Fuzzy Trace Theory (Deese, 1959; Reyna \& Brainerd, 1995; Roediger \& McDermott, 1995b), which posits that

\footnotetext{
${ }^{2}$ This result should be interpreted with caution as true compared to false familiarity did show greater activation in late visual cortex (BA 19), though the cluster (51 voxels) fell shy of the corrected threshold (66 voxels).
} 
gist traces support false memories, particularly in the absence of verbatim traces that offset the familiarity of the lure item.

As noted, prior false memory studies have disagreed about the role of the MTL in mediating false memories (see Dennis et al., 2015 for review). That is, some studies find the MTL to be active for both true and false retrieval (Cabeza et al., 2001; Garoff-Eaton et al., 2006; Kahn et al., 2004; Schacter et al., 1997; Schacter et al., 1996; Stark et al., 2010), whereas others have found MTL activity only for retrieval of true, but not false, memories (Dennis et al., 2008b; Giovanello, Kensinger, Wong, \& Schacter, 2009; Kim \& Cabeza, 2007b; Paz-Alonso, Ghetti, Donohue, Goodman, \& Bunge, 2008). In our earlier work (Dennis et al., 2012) we posited that this discrepancy might be due, in part, to inconsistencies in testing procedures and the tendency of past studies to collapse across recollection- and familiarity-related responses. Supporting this theory, as well as our predictions, the current findings showed the MTL elicited different patterns of activity when comparing true and false memories across recollection and familiarity. Specifically, bilateral anterior hippocampus showed greater activity for schematic true compared to false recollection, while schematic false compared to true familiarity exhibited greater activity in a more posterior region of left hippocampus/PHG (see Figure 3B).

Regarding recollection, the current results support our earlier work, as well as that of others, who have separated out recollection processes with respect to true and false memories, identifying greater activity for true recollection in the hippocampus proper (Abe et al., 2013; Dennis et al., 2012; Kim \& Cabeza, 2007b). Similar to the interpretation of greater MTL activity for schematic compared to nonschematic recollection above, current results suggest that true recollection is accompanied by greater recovery of item-specific episodic details from encoding, as well as the binding of multiple memory traces that is unique to recollection (Diana et al., 2007; Yonelinas et al., 2005).

While familiarity is generally defined as the absence of vivid retrieval for item-specific details, the current results showed false familiarity to be mediated by activity in left hippocampus/PHG. The current MTL results are consistent with recent false memory work that has broken down false recollection from that of familiarity, showing greater PHG activity for false compared to true familiarity (Abe et al., 
2013; Karanian \& Slotnick, 2014; see also Kim \& Cabeza, 2007b). Despite the apparent dichotomy, a parsimonious explanation has been offered by Karanian and Slotnick (2014). Specifically, they suggest that within the context of false familiarity, PHG activity reflects a greater reliance on source processing in the face of a weak memory trace. That is, when only a weak retrieval trace is available, (such as only a low level of gist), false familiarity is supported by retrieval of the encoding source or surrounding details. For example, in the current study, participants may be recollecting the contextual event (i.e., gist of the scene), but are still lacking details of the item itself and are thus indicating a familiarity response.

Interestingly, non-schematic familiarity also exhibited significantly greater recruitment of this region compared to schematic familiarity, and showed no difference with respect to schematic false familiarity. This result further supports the aforementioned conclusion that, when faced with a relatively weak item trace, enhanced retrieval of the encoding context may underlie a familiarity response for a given item. Further research should continue to build upon these theories of MTL involvement in true and false retrieval, specifically with respect to the separate contributions of recollection and familiarity processing.

4.5. Conclusions. Schemas have been shown to be both beneficial to memory, supporting encoding and retrieval success, but also detrimental when novel information is highly related to the schema. The current study sought to investigate the influence that a schematic framework at encoding has on both veridical and false memories across different levels of memory strength. In doing so, the study built on recent work that has highlighted the need to examine the separate contributions of recollection and familiarity in the context of false memories (Abe et al., 2013; Dennis et al., 2012). Furthermore, the use of the scene paradigm allowed us to investigate the neural basis of false memory for pictorial items that have a conceptual, rather than a perceptual, relationship with encoded items. The study identified several novel results. In terms of memory success, while we identified a wealth of common regions supporting both schematic and non-schematic memory success, schematic recollection was supported by detailed retrieval mediated by the hippocampus and visual cortex. The more difficult non-schematic recollection, however, relied on regions supporting more effortful retrieval monitoring and evaluation strategies. With respect to 
false memories, common retrieval activity across veridical and illusory memory was found in lateral temporal regions that support semantic processing and retrieval of schematic gist. Moreover, activity in this region was greater for both false recollection and false familiarity suggesting that retrieval schematic information from encoding was a critical factor in mediating illusory memories, irrespective of memory strength. Importantly, the absence of common visual activity, suggests that, unlike previous studies utilizing visual stimuli, when false memories are predicated on schematic gist and not perceptual overlap, there is little reliance on visual processes during false memory retrieval. Furthermore, as visual activity was greater for true recollection, results suggest that visual cortices are critical for distinguishing true and false memories. Finally, the dissociation identified within the MTL suggests that memory strength is a critical factor to understanding the role of this region in supporting true and false memories. This study builds on past fMRI false memory research, clarifying and expanding our knowledge of the neural mechanisms supporting memory. 


\section{Acknowledgements}

The authors wish to thank Courtney Allen for help with stimulus development, Kristina Peterson for assistance in data collection, and Caitlin Bowman, John Huhn, and Kyle Kurkela for comments on earlier versions of the manuscript. We also wish to thank the Penn State Social, Life, \& Engineering Sciences Imaging Center (SLEIC), 3T MRI Facility. This work was supported by a National Science Foundation grant (BCS1025709) awarded to NAD. CEW was partially supported by National Institute on Aging Grant T32 (AG049676) awarded to The Pennsylvania State University and ICT was supported by a Graduate Research Fellowship from the National Science Foundation (DGE1255832). Any opinions, findings, and conclusions or recommendations expressed in this material are those of the author(s) and do not necessarily reflect the views of the National Science Foundation or National Institute on Aging. 


\section{Table 1. Behavioral Results}

Collapsed Response Rates

Hit

FA
Schematic

$.69(.02)$

$.54(.03)$

$.42(.06)$

$.44(.03)$

$.22(.02)$

$.25(.02) / .45(.03)$

$.32(.02) / .41(.03)$
Non-Schematic

$.54(.03)$

$.24(.04)$

$.92(.08)$

Recollection Response Rates

Hit

Familiarity Response Rates

Hit / Adjusted Hit

FA / Adjusted FA

The table reports the

means and standard errors of the proportion of Collapsed

(Recollection/'Remember' and Familiarity/'Know') response rates and for schematic and non-schematic targets (Hits) and lures (False Alarms; FA), as well as d' values.

Both raw and adjusted familiarity responses are reported. Adjusted familiarity hits are calculated as pKnow Hits/(1 - pRemember Hits) and adjusted familiarity FA are calculated as pKnow FA/(1 - pRemember FA). 
Table 2. Common Schematic and Non-schematic Retrieval Success Activity

\begin{tabular}{|c|c|c|c|c|c|c|c|}
\hline & BA & $\mathrm{H}$ & & $\begin{array}{l}\text { rdina } \\
\Gamma \& T)\end{array}$ & & $t$ & $\mathrm{~mm}^{3}$ \\
\hline & & & $\mathrm{X}$ & $\mathrm{Y}$ & $\mathrm{Z}$ & & \\
\hline$\underline{\text { Recollection }}$ & & & & & & & \\
\hline$\overline{\text { Middle Frontal Gyrus }}$ & $10 / 11$ & $\mathrm{~L}$ & -39 & 51 & -7 & 5.34 & 5076 \\
\hline & $46 / 9$ & $\mathrm{~L}$ & -48 & 29 & 22 & 5.37 & 7749 \\
\hline & 9 & $\mathrm{R}$ & 42 & 12 & 33 & 5.03 & 4320 \\
\hline Caudate & - & $\mathrm{R}$ & 9 & 11 & 3 & 5.1 & 2619 \\
\hline & - & $\mathrm{L}$ & -9 & 11 & 0 & 5.23 & 4401 \\
\hline Middle Temporal Gyrus & 21 & $\mathrm{~L}$ & -59 & -36 & -7 & 5.01 & 11718 \\
\hline & & $\mathrm{R}$ & 59 & -36 & -7 & 4.55 & 6264 \\
\hline Inferior/Superior Parietal Lobe & $40 / 7$ & $\mathrm{~L}$ & -33 & -51 & 45 & 6.78 & 29646 \\
\hline & & $\mathrm{R}$ & 36 & -60 & 48 & 6.02 & 14391 \\
\hline$\underline{\text { Familiarity }}$ & & & & & & & \\
\hline Middle Frontal Gyrus & $46 / 9$ & $\mathrm{~L}$ & -45 & 35 & 21 & 4.18 & 3375 \\
\hline Inferior Frontal Gyrus & 10 & $\mathrm{~L}$ & -45 & 46 & 4 & 4.38 & 3213 \\
\hline Middle Temporal Gyrus & 21 & $\mathrm{~L}$ & -50 & -36 & 3 & 4.13 & 1998 \\
\hline Superior Parietal Lobe & 7 & $\mathrm{~L}$ & -30 & -51 & 45 & 4.09 & 3105 \\
\hline
\end{tabular}

Commonly activated regions for schematic and non-schematic recollection and familiarity retrieval success. $\mathrm{BA}=$ Brodmann's Area; $\mathrm{H}=$ Hemisphere; $\mathrm{L}=$ Left; $\mathrm{M}=$ Medial; $\mathrm{R}=$ Right; $\mathrm{t}=$ statistical $\mathrm{t}$-value;

$\mathrm{T} \& \mathrm{~T}=$ Talairach and Tournoux coordinates.

Italics represent a priori ROIs. 
Table 3. Schema Differences in Target Retrieval

\begin{tabular}{ccccccc} 
BA & $\mathrm{H}$ & \multicolumn{3}{c}{ Coordinates (T\&T) } & $t$ & $\mathrm{~mm}^{3}$ \\
& & $\mathrm{X}$ & $\mathrm{Y}$ & $\mathrm{Z}$ & & \\
& & & & & & \\
& & & & & & \\
- & $\mathrm{L}$ & -27 & -13 & -9 & 4.28 & 648 \\
19 & $\mathrm{~L}$ & -48 & -76 & 8 & 5.85 & 3807 \\
$19 / 37$ & $\mathrm{R}$ & 50 & -71 & 4 & 5.75 & 6669
\end{tabular}

Familiarity

No suprathreshold clusters

\title{
$\underline{\text { Non-schematic }>\text { Schematic }}$
}

\author{
Recollection
}

Anterior Cingulate

Supramarginal Gyrus

$\begin{array}{ll}\mathrm{L} & -6\end{array}$

$\begin{array}{lll}40 & \mathrm{~L} & -42\end{array}$

\section{0}

27

3.75

2457

\section{Familiarity}

Middle Temporal Gyrus

$\begin{array}{lccccc}\mathrm{L} & -56 & -16 & -19 & 4.88 & 1296 \\ \mathrm{R} & 56 & -19 & -13 & 3.93 & 945\end{array}$

Areas exhibiting differential activation for schematic and non-schematic recollection and familiarity. BA = Brodmann's Area; $\mathrm{H}=$ Hemisphere; $\mathrm{L}=$ Left; $\mathrm{M}=$ Medial; $\mathrm{R}=$ Right; $\mathrm{t}=$ statistical $\mathrm{t}$-value; $\mathrm{T} \& \mathrm{~T}=$ Talairach and Tournoux coordinates. Italics represent a priori ROIs. 


\section{Table 4. Common True and False Memory Activity}

\begin{tabular}{|c|c|c|c|c|c|c|c|}
\hline \multirow{2}{*}{ Doullotion } & \multirow[t]{2}{*}{ BA } & \multirow[t]{2}{*}{$\mathrm{H}$} & \multicolumn{3}{|c|}{ Coordinates $(\mathrm{T} \& \mathrm{~T})$} & \multirow[t]{2}{*}{$t$} & \multirow[t]{2}{*}{$\mathrm{mm}^{3}$} \\
\hline & & & $\mathrm{X}$ & $\mathrm{Y}$ & $\mathrm{Z}$ & & \\
\hline Middle Frontal Gyrus & $10 / 46$ & $\mathrm{~L}$ & -42 & 45 & -4 & 4.57 & 2997 \\
\hline Inferior Parietal Lobe & $7 / 40$ & $\mathrm{~L}$ & -33 & -57 & 42 & 4.67 & 4428 \\
\hline Inferior Parietal Lobe/Superior Occipital Gyrus & $19 / 39$ & $\mathrm{R}$ & 39 & -72 & 35 & 5.22 & 2079 \\
\hline Middle Temporal Gyrus & 21 & $\mathrm{~L}$ & -50 & -33 & 0 & 4.26 & 972 \\
\hline
\end{tabular}

Commonly activated regions for true and false recollection and true and false familiarity. BA = Brodmann's Area; $\mathrm{H}=$ Hemisphere; $\mathrm{L}=$ Left; $\mathrm{M}=$ Medial; $\mathrm{R}=$ Right; $\mathrm{t}=$ statistical $\mathrm{t}$-value; $\mathrm{T} \& \mathrm{~T}=$ Talairach and Tournoux coordinates. Italics represent a priori ROIs. 


\section{Table 5. Schematic True and False Memory Differences}

BA $\quad \mathrm{H} \quad \mathrm{X} \quad \mathrm{Y} \quad \mathrm{Z} \quad \mathrm{mm}^{3}$

\section{$\underline{\text { True }>\text { False }}$}

\section{$\underline{\text { Recollection }}$}

Hippocampus

Middle Temporal Gyrus

Middle/Superior Temporal Gyrus

Inferior/Middle Occipital Gyrus

Cerebellum

-
21
$21 / 22$
$18 / 19$

R 24

$-2$

$-17$

4.06

$\begin{array}{lll}\mathrm{L} & -33 & -11\end{array}$

$-28$

$-16$

4.15

1296

$\mathrm{R} \quad 59 \quad-28$

$\mathrm{R} \quad 59$

$-48$

$-79$

5.84

11151

-

L $\quad-27$

3.60

Familiarity

No suprathreshold voxels

\section{False $>$ True}

$\underline{\text { Recollection }}$

Superior Temporal Gyrus

$$
41 / 42
$$

$41 / 42$

R $\quad 45$

$$
-29
$$

16

4.66

1188

Familiarity

Hippocampus/PHG

Middle/Superior Temporal Gyrus

Middle Temporal Gyrus

$\begin{array}{ccccccc}- & \mathrm{L} & -33 & -28 & -10 & 4.2 & 1593 \\ 21 / 20 / 22 & \mathrm{R} & 59 & -19 & -8 & 5.22 & 5454 \\ 21 / 20 / 22 & \mathrm{~L} & -59 & -39 & -7 & 4.94 & 7911 \\ 20 & \mathrm{R} & 48 & 4 & -27 & 3.82 & 675\end{array}$

Areas exhibiting differential activation for true and false recollection and familiarity. BA = Brodmann's Area; $\mathrm{H}=$ Hemisphere; $\mathrm{L}=$ Left; $\mathrm{M}=$ Medial; $\mathrm{R}=$ Right; $\mathrm{t}=$ statistical $\mathrm{t}$-value; $\mathrm{T} \& \mathrm{~T}=$ Talairach and Tournoux coordinates. Italics represent a priori ROIs. 


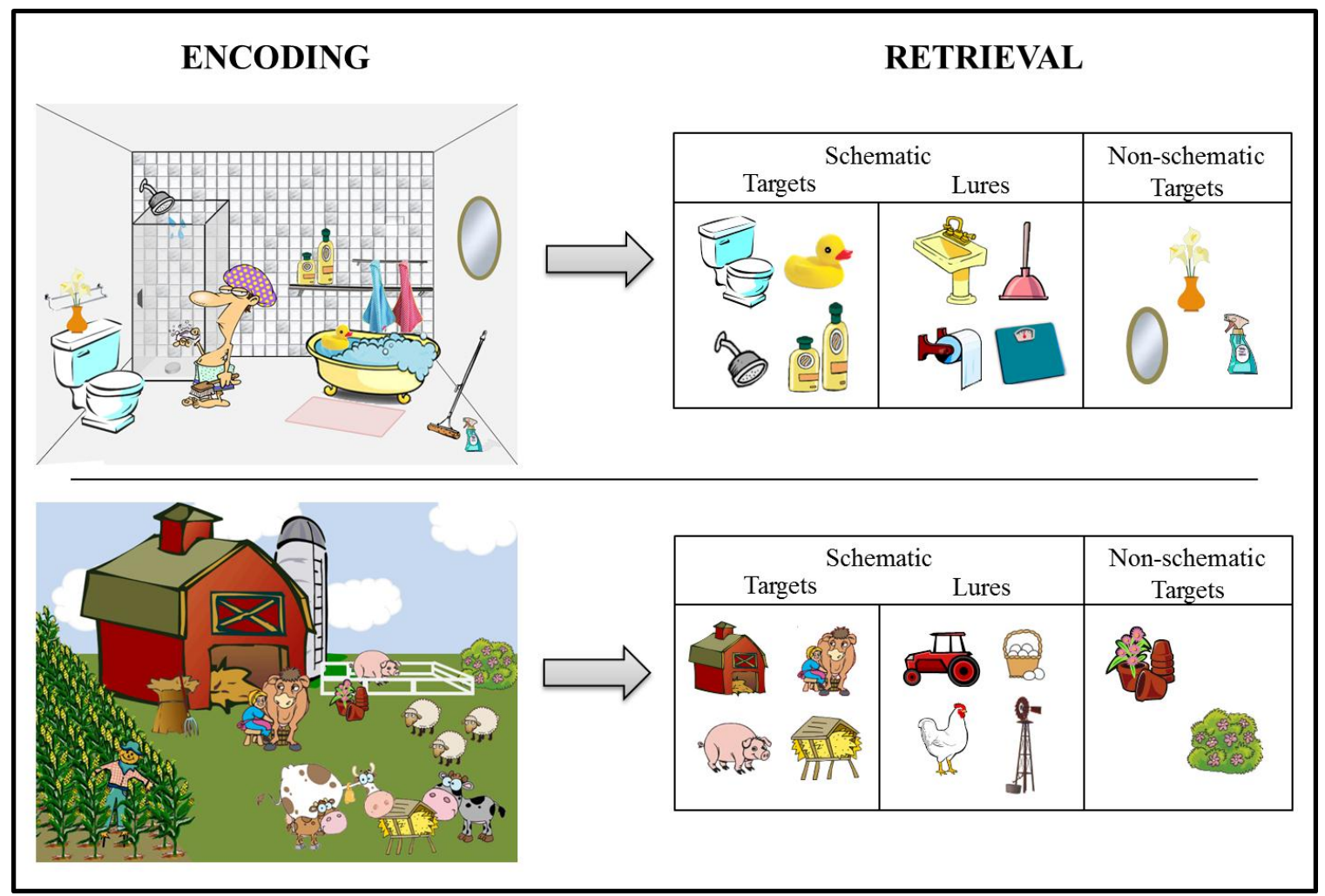

Figure 1. Task Design. During encoding, participants viewed schematic scenes (e.g., Bathroom, Farm). During retrieval, participants made 'Remember', 'Know', or 'New' responses to both schematicallyrelated targets (e.g., toilet, pig) and schematically-related lures (e.g., sink, tractor), as well as nonschematic targets and lures. 


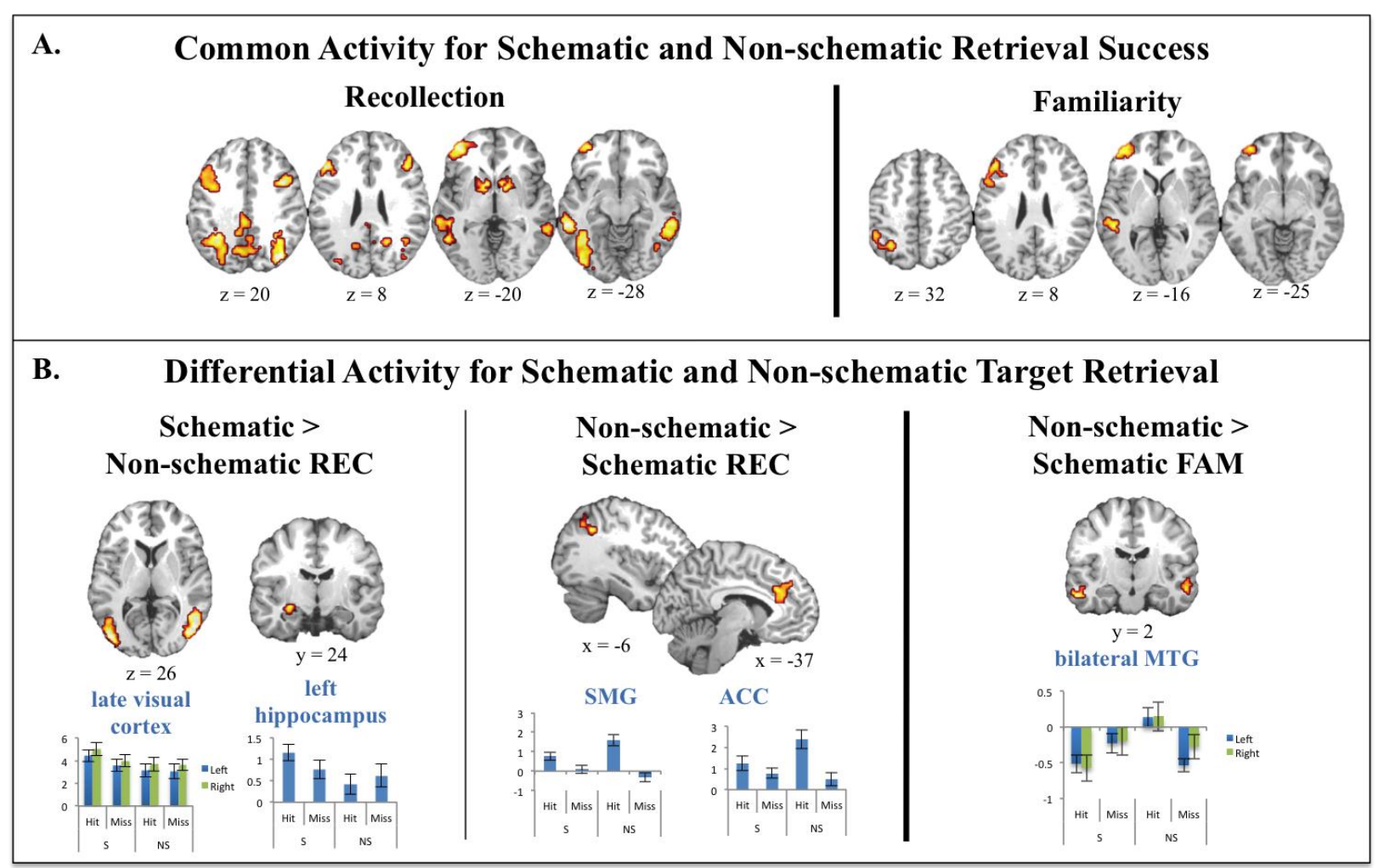

Figure 2. Schematic and Non-schematic Memory. A) Regions showing common activity for schematic and non-schematic recollection- and familiarity-based retrieval success B) Regions showing differential activity for schematic and non-schematic recollection and familiarity. Y-axes represent beta estimates. $\mathrm{REC}=$ Recollection; FAM = Familiarity; $\mathrm{S}=$ Schematic; $\mathrm{NS}=$ Non-schematic; $\mathrm{SMG}=$ Supramarginal Gyrus; $\mathrm{ACC}=$ Anterior Cingulate Cortex; $\mathrm{MTG}=$ Middle Temporal Gyrus . 


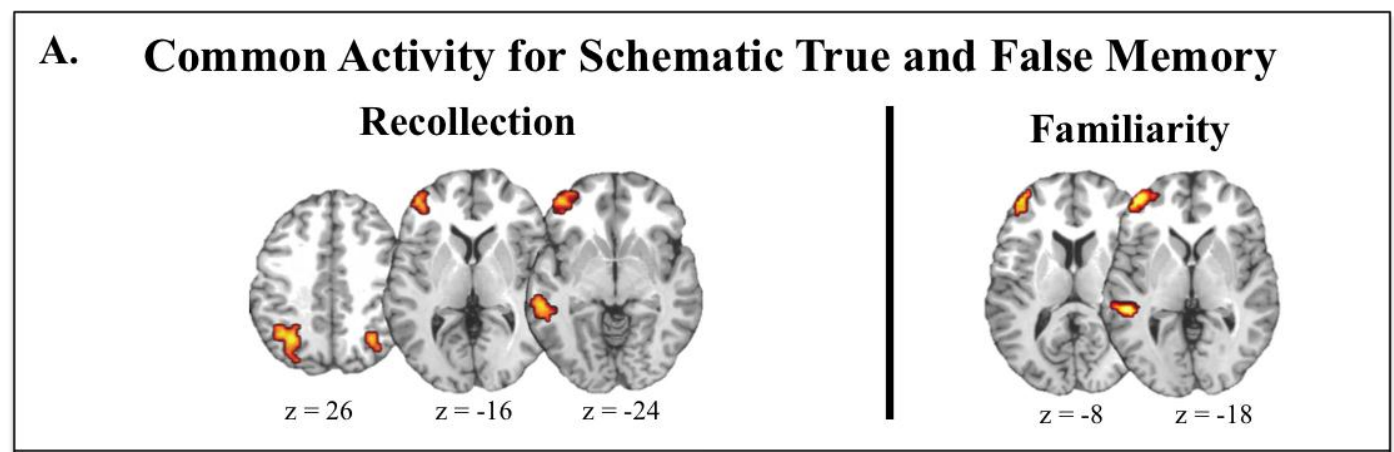

\section{B. Differential Activity for Schematic True and False Memory}

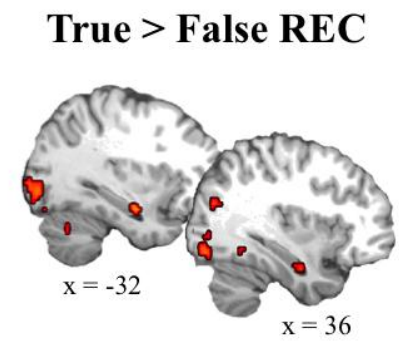

bilateral hippocampus

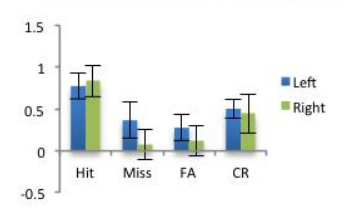

False $>$ True REC

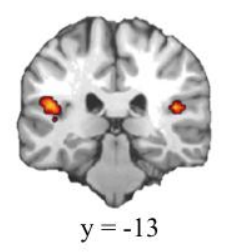

bilateral STG

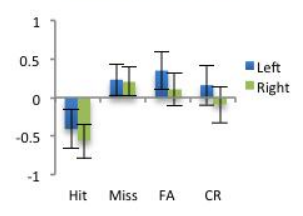

False $>$ True FAM

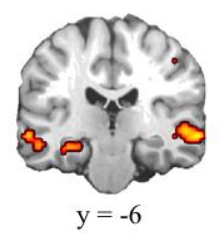

bilateral MTG/STG and left hippocampus/PHG

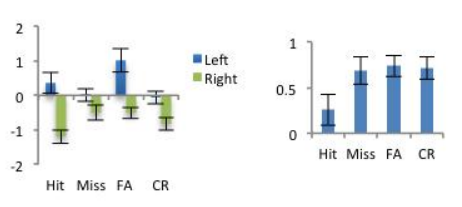

Figure 3. True and False Memory. A) Regions showing common activity for true and false recollection and familiarity B) Regions showing differential activity for true and false recollection and familiarity. Yaxis represents beta estimates. $\mathrm{REC}=$ Recollection; FAM = Familiarity; $\mathrm{S}=$ Schematic; NS = Nonschematic; FA = False Alarm; CR = Correct Rejection; STG = Superior Temporal Gyrus; MTG = Middle Temporal Gyrus; PHG = Parahippocampal Gyrus. 


\section{References}

Abe, N., Fujii, T., Suzuki, M., Ueno, A., Shigemune, Y., Mugikura, S., . . Mori, E. (2013). Encodingand retrieval-related brain activity underlying false recognition. Neurosci Res, 76(4), 240-250.

Alba, J. W., \& Hasher, L. (1983). Is Memory Schematic? Psychological Bulletin, 93(2), 203-231. doi: Doi 10.1037/0033-2909.93.2.203

Aminoff, E., Schacter, D. L., \& Bar, M. (2008). The cortical underpinnings of context-based memory distortion. Journal of Cognitive Neuroscience, 20(12), 2226-2237.

Atkins, A. S., \& Reuter-Lorenz, P. A. (2011). Neural mechanisms of semantic interference and false recognition in short-term memory. Neuroimage, 56(3), 1726-1734.

Bar, M., \& Aminoff, E. (2003). Cortical analysis of visual context. Neuron, 38(2), 347-358.

Bar, M., Aminoff, E., \& Schacter, D. L. (2008). Scenes unseen: the parahippocampal cortex intrinsically subserves contextual associations, not scenes or places per se. J Neurosci, 28(34), 8539-8544. doi: 10.1523/JNEUROSCI.0987-08.2008

Binder, J. R., Desai, R. H., Graves, W. W., \& Conant, L. L. (2009). Where is the semantic system? A critical review and meta-analysis of 120 functional neuroimaging studies. Cereb Cortex, 19(12), 2767-2796. doi: 10.1093/cercor/bhp055

Bower, G. H., Black, J. B., \& Turner, T. J. (1979). Scripts in Memory for Text. Cognitive Psychology, 11(2), 177-220.

Brainerd, C. J., Wright, R., Reyna, V. F., \& Mojardin, A. H. (2001). Conjoint recognition and phantom recollection. Journal of Experimental Psycholgy: Learning, Memory, \& Cognition, 27(2), 307327.

Brewer, W. F., \& Treyens, J. C. (1981). Role of schemata in memory for places. Cognitive Psychology, 13, 207-230.

Buckner, R. L. (1996). Beyond HERA: Contributions of specific prefrontal brain areas to long-term memory retrieval. Psychon Bull Rev, 3(2), 149-158. doi: Doi 10.3758/Bf03212413

Buckner, R. L., \& Wheeler, M. E. (2001). The cognitive neuroscience of remembering. Nat Rev Neurosci, 2(9), 624-634.

Cabeza, R., Ciaramelli, E., Olson, I. R., \& Moscovitch, M. (2008). The parietal cortex and episodic memory: an attentional account. Nat Rev Neurosci, 9(8), 613-625.

Cabeza, R., Rao, S. M., Wagner, A. D., Mayer, A. R., \& Schacter, D. L. (2001). Can medial temporal lobe regions distinguish true from false? An event-related functional MRI study of veridical and illusory recognition memory. Proc Natl Acad Sci U S A, 98(8), 4805-4810.

Carter, C. S., Braver, T. S., Barch, D. M., Botvinick, M. M., Noll, D., \& Cohen, J. D. (1998). Anterior cingulate cortex, error detection, and the online monitoring of performance. Science, 280(5364), 747-749.

Ciaramelli, E., Grady, C., Levine, B., Ween, J., \& Moscovitch, M. (2010). Top-down and bottom-up attention to memory are dissociated in posterior parietal cortex: neuroimagingand and neuropsychological evidence. $J$ Neurosci, 30(14), 4943-4956. doi: 10.1523/JNEUROSCI.120909.2010

Ciaramelli, E., Grady, C. L., \& Moscovitch, M. (2008). Top-down and bottom-up attention to memory: a hypothesis (AtoM) on the role of the posterior parietal cortex in memory retrieval. Neuropsychologia, 46(7), 1828-1851. doi: 10.1016/j.neuropsychologia.2008.03.022

Cox, R. W., \& Hyde, J. S. (1997). Software tools for analysis and visualization of fMRI data. Nmr in Biomedicine, 10(4-5), 171-178. doi: Doi 10.1002/(Sici)1099-1492(199706/08)10:4/5<171::AidNbm453>3.0.Co;2-L

Daselaar, S. M., Fleck, M. S., \& Cabeza, R. E. (2006). Triple Dissociation in the Medial Temporal Lobes: Recollection, Familiarity, and Novelty. J Neurophysiol, 96, 1902-1911. 
Deese, J. (1959). On the prediction of occurrence of particular verbal intrusions in immediate recall. Journal of Experimental Psychology, 58(1), 17-22.

Dennis, N. A., Bowman, C. R., \& Peterson, K. P. (2014). Age-related differences in the neural correlates mediating false recollection. Neurobiology of Aging, 35, 395-407.

Dennis, N. A., Bowman, C. R., \& Turney, I. C. (2015). Functional Neuroimaging of False Memories. In D. R. Addis, M. Barense \& A. Duarte (Eds.), The Wiley Handbook on the Cognitive Neuroscience of Memory. Chichester, UK: John Wiley \& Sons, Ltd.

Dennis, N. A., Bowman, C. R., \& Vandekar, S. N. (2012). True and phantom recollection: an fMRI investigation of similar and distinct neural correlates and connectivity. Neuroimage, 59(3), 29822993.

Dennis, N. A., Kim, H., \& Cabeza, R. (2008b). Age-related differences in brain activity during true and false memory retrieval. Journal of Cognitive Neuroscience, 20(8), 1390-1402.

Dennis, N. A., Kim, H. K., \& Cabeza, R. (2007b). Effects of aging on the neural correlates of true and false memory formation. Neuropsychologia, 45, 3157-3166.

Diana, R. A., Yonelinas, A. P., \& Ranganath, C. (2007). Imaging recollection and familiarity in the medial temporal lobe: a three-component model. Trends Cogn Sci, 11(9), 379-386.

Duarte, A., Graham, K. S., \& Henson, R. N. (2010). Age-related changes in neural activity associated with familiarity, recollection and false recognition. Neurobiology of Aging, 31(10), 1814-1830.

Duarte, A., Ranganath, C., Trujillo, C., \& Knight, R. T. (2006). Intact recollection memory in highperforming older adults: ERP and behavioral evidence. Journal of Cognitive Neuroscience, 18(1), 33-47. doi: 10.1162/089892906775249988

Eichenbaum, H., Sauvage, M., Fortin, N., Komorowski, R., \& Lipton, P. (2012). Towards a functional organization of episodic memory in the medial temporal lobe. Neurosci Biobehav Rev, 36(7), 1597-1608.

Eldridge, L. L., Knowlton, B. J., Furmanski, C. S., Bookheimer, S. Y., \& Engel, S. A. (2000). Remembering episodes: a selective role for the hippocampus during retrieval. Nat Neurosci, 3(11), 1149-1152.

Fleck, M. S., Daselaar, S. M., Dobbins, I. G., \& Cabeza, R. (2006). Role of Prefrontal and Anterior Cingulate Regions in Decision-Making Processes Shared by Memory and Nonmemory Tasks. Cereb Cortex.

Gabrieli, J. D., Poldrack, R. A., \& Desmond, J. E. (1998). The role of left prefrontal cortex in language and memory. Proc Natl Acad Sci US A, 95(3), 906-913.

Garoff-Eaton, R. J., Kensinger, E. A., \& Schacter, D. L. (2007). The neural correlates of conceptual and perceptual false recognition. Learn Mem, 14(10), 684-692.

Garoff-Eaton, R. J., Slotnick, S. D., \& Schacter, D. L. (2006). Not all false memories are created equal: the neural basis of false recognition. Cereb Cortex, 16(11), 1645-1652.

Giovanello, K. S., Kensinger, E. A., Wong, A. T., \& Schacter, D. L. (2009). Age-related neural changes during memory conjunction errors. Journal of Cognitive Neuroscience, 22(7), 1348-1361.

Goldmann, R. E., Sullivan, A. L., Droller, D. B., Rugg, M. D., Curran, T., Holcomb, P. J., . . Budson, A. E. (2003). Late frontal brain potentials distinguish true and false recognition. Neuroreport, 14(13), 1717-1720.

Gutchess, A. H., \& Schacter, D. L. (2012). The neural correlates of gist-based true and false recognition. Neuroimage, 59(4), 3418-3426. doi: 10.1016/j.neuroimage.2011.11.078

Hannigan, S. L., \& Reinitz, M. T. (2003). Migration of objects and inferences across episodes. Mem Cognit, 31(3), 434-444.

Hess, T. M., \& Slaughter, S. J. (1990). Semantic knowledge influences on memory for scene information in young and older adults. Dev Psychol, 26(5), 855-865.

Iidaka, T., Harada, T., Kawaguchi, J., \& Sadato, N. (2012). Neuroanatomical substrates involved in true and false memories for face. Neuroimage, 62(1), 167-176. 
Intraub, H., Gottesman, C. V., Willey, E. V., \& Zuk, I. J. (1996). Boundary extension for briefly glimpsed photographs: Do common perceptual processes result in unexpected memory distortions? Journal of Memory and Language, 35(2), 118-134. doi: DOI 10.1006/jmla.1996.0007

Kahn, I., Davachi, L., \& Wagner, A. D. (2004). Functional-neuroanatomic correlates of recollection: implications for models of recognition memory. Journal of Neuroscience, 24(17), 4172-4180.

Karanian, J. M., \& Slotnick, S. D. (2014). False memory for context activates the parahippocampal cortex. Cogn Neurosci, 5(3-4), 186-192.

Kim, H. K., \& Cabeza, R. (2007b). Trusting our memories: Dissociating the neural correlates of confidence in veridical and illusory memories. Journal of Neuroscience, 27, 12190-12197.

Kircher, T. T., Brammer, M., Tous Andreu, N., Williams, S. C., \& McGuire, P. K. (2001). Engagement of right temporal cortex during processing of linguistic context. Neuropsychologia, 39(8), 798-809.

Kubota, Y., Toichi, M., Shimizu, M., Mason, R. A., Findling, R. L., Yamamoto, K., \& Calabrese, J. R. (2006). Prefrontal hemodynamic activity predicts false memory--a near-infrared spectroscopy study. Neuroimage, 31(4), 1783-1789.

Kurkela, K. A., \& Dennis, N. A. (2016). Event-related fMRI studies of false memory: An Activation Likelihood Estimation meta-analysis. Neuropsychologia, 81, 149-167. doi: 10.1016/j.neuropsychologia.2015.12.006

Lampinen, J. M., Copeland, S. M., \& Neuschatz, J. S. (2001). Recollections of things schematic: room schemas revisited. Journal of Experimental Psychology: Learning, Memory, \& Cognition, 27(5), 1211-1222.

Lampinen, J. M., Faries, J. M., Neuschatz, J. S., \& Toglia, M. P. (2000). Recollections of things schematic: The influence of scripts on recollective experience. Applied Cognitive Psychology, 14(6), 543-554.

Lancaster, J. L., Woldorff, M. G., Parsons, L. M., Liotti, M., Freitas, C. S., Rainey, L., . . Fox, P. T. (2000). Automated Talairach atlas labels for functional brain mapping. Human Brain Mapping, 10(3), 120-131.

Loftus, G. R., \& Mackworth, N. H. (1978). Cognitive Determinants of Fixation Location during Picture Viewing. Journal of Experimental Psychology-Human Perception and Performance, 4(4), 565572.

Maldjian, J. A., Laurienti, P. J., Kraft, R. A., \& Burdette, J. H. (2003). An automated method for neuroanatomic and cytoarchitectonic atlas-based interrogation of fMRI data sets. Neuroimage, 19(3), 1233-1239.

Mather, M., Henkel, L. A., \& Johnson, M. K. (1997). Evaluating characteristics of false memories: remember/know judgments and memory characteristics questionnaire compared. Mem Cognit, 25(6), 826-837.

McCabe, D. P., Roediger, H. L., 3rd, McDaniel, M. A., \& Balota, D. A. (2009). Aging reduces veridical remembering but increases false remembering: neuropsychological test correlates of rememberknow judgments. Neuropsychologia, 47(11), 2164-2173.

Miller, M. B., \& Gazzaniga, M. S. (1998). Creating false memories for visual scenes. Neuropsychologia, 36(6), 513-520.

Neuschatz, J. S., Lampinen, J. M., Preston, E. L., Hawkins, E. R., \& Toglia, M. P. (2002). The effect of memory schemata on memory and the phenomenological experience of naturalistic situations. Applied Cognitive Psychology, 16(6), 687-708.

Noppeney, U., Patterson, K., Tyler, L. K., Moss, H., Stamatakis, E. A., Bright, P., . . Price, C. J. (2007). Temporal lobe lesions and semantic impairment: a comparison of herpes simplex virus encephalitis and semantic dementia. Brain, 130(Pt 4), 1138-1147.

Noppeney, U., \& Price, C. J. (2002). A PET study of stimulus- and task-induced semantic processing. Neuroimage, 15(4), 927-935. doi: 10.1006/nimg.2001.1015

Norman, K. A., \& Schacter, D. L. (1997). False recognition in younger and older adults: exploring the characteristics of illusory memories. Mem Cognit, 25(6), 838-848. 
Oliva, A., \& Torralba, A. (2001). Modeling the shape of the scene: A holistic representation of the spatial envelope. International Journal of Computer Vision, 42(3), 145-175.

Owens, J., Bower, G. H., \& Black, J. B. (1979). The "soap opera" effect in story recall. Mem Cognit, 7(3), 185-191.

Paz-Alonso, P. M., Ghetti, S., Donohue, S. E., Goodman, G. S., \& Bunge, S. A. (2008). Neurodevelopmental correlates of true and false recognition. Cereb Cortex, 18(9), 2208-2216.

Pezdek, K., Whetstone, T., Reynolds, K., Askari, N., \& Dougherty, T. (1989). Memory for Real-World Scenes - the Role of Consistency with Schema Expectation. Journal of Experimental PsychologyLearning Memory and Cognition, 15(4), 587-595.

Potter, M. C. (1976). Short-term conceptual memory for pictures. J Exp Psychol Hum Learn, 2(5), 509522.

Price, C. J. (2000). The anatomy of language: contributions from functional neuroimaging. $J$ Anat, 197 Pt $3,335-359$.

Ranganath, C. (2010). Binding Items and Contexts: The Cognitive Neuroscience of Episodic Memory. Current Directions in Psychological Science, 19(3), 131-137.

Reyna, V. F., \& Brainerd, C. J. (1995). Fuzzy-Trace Theory - an Interim Synthesis. Learning and Individual Differences, 7(1), 1-75.

Roediger, H. L., 3rd, \& Mcdermott, K. B. (1995a). Creating False Memories - Remembering Words Not Presented in Lists. Journal of Experimental Psychology-Learning Memory and Cognition, 21(4), 803-814.

Roediger, H. L., 3rd, \& McDermott, K. B. (1995b). Creating false memories: Remembering words not presented in lists. Journal of Experimental Psychology: Learning, Memory and Cognition, 21, 8033-8814.

Rugg, M. D., \& Vilberg, K. L. (2013). Brain networks underlying episodic memory retrieval. Curr Opin Neurobiol, 23(2), 255-260. doi: 10.1016/j.conb.2012.11.005

Rugg, M. D., \& Wilding, E. L. (2000). Retrieval processing and episodic memory. Trends Cogn Sci, 4(3), 108-115.

Saumier, D., \& Chertkow, H. (2002). Semantic memory. Current neurology and neuroscience reports, 2(6), 516-522.

Schacter, D. L., Buckner, R. L., Koutstaal, W., Dale, A. M., \& Rosen, B. R. (1997). Late onset of anterior prefrontal activity during true and false recognition: an event-related fMRI study. Neuroimage, 6(4), 259-269.

Schacter, D. L., Reiman, E., Curran, T., Yun, L. S., Bandy, D., McDermott, K. B., \& Roediger, H. L., 3rd. (1996). Neuroanatomical correlates of veridical and illusory recognition memory: evidence from positron emission tomography. Neuron, 17(2), 267-274.

Simons, J. S., Verfaellie, M., Hodges, J. R., Lee, A. C., Graham, K. S., Koutstaal, W., . . Budson, A. E. (2005). Failing to get the gist: reduced false recognition of semantic associates in semantic dementia. Neuropsychology, 19(3), 353-361.

Slotnick, S. D., \& Schacter, D. L. (2004). A sensory signature that distinguishes true from false memories. Nat Neurosci, 7(6), 664-672.

Slotnick, S. D., \& Schacter, D. L. (2006). The nature of memory related activity in early visual areas. Neuropsychologia, 44(14), 2874-2886.

Spaniol, J., Davidson, P. S., Kim, A. S., Han, H., Moscovitch, M., \& Grady, C. L. (2009). Event-related fMRI studies of episodic encoding and retrieval: meta-analyses using activation likelihood estimation. Neuropsychologia, 47(8-9), 1765-1779. doi: 10.1016/j.neuropsychologia.2009.02.028

Stark, C. E., Okado, Y., \& Loftus, E. F. (2010). Imaging the reconstruction of true and false memories using sensory reactivation and the misinformation paradigms. Learn Mem, 17(10), 485-488.

Talairach, J., \& Tournoux, P. (1988). Co-planar stereotaxic atlas of the human brain. Stuttgart, Germany: Thieme. 
Torralba, A., Oliva, A., Castelhano, M. S., \& Henderson, J. M. (2006). Contextual guidance of eye movements and attention in real-world scenes: The role of global features in object search. Psychological Review, 113(4), 766-786.

Tse, D., Langston, R. F., Kakeyama, M., Bethus, I., Spooner, P. A., Wood, E. R., . . Morris, R. G. (2007). Schemas and memory consolidation. Science, 316(5821), 76-82.

Tse, D., Takeuchi, T., Kakeyama, M., Kajii, Y., Okuno, H., Tohyama, C., . . Morris, R. G. (2011). Schema-dependent gene activation and memory encoding in neocortex. Science, 333(6044), 891 895. doi: $10.1126 /$ science. 1205274

Vaidya, C. J., Zhao, M., Desmond, J. E., \& Gabrieli, J. D. (2002). Evidence for cortical encoding specificity in episodic memory: memory-induced re-activation of picture processing areas. Neuropsychologia, 40(12), 2136-2143.

van Buuren, M., Kroes, M. C., Wagner, I. C., Genzel, L., Morris, R. G., \& Fernandez, G. (2014). Initial investigation of the effects of an experimentally learned schema on spatial associative memory in humans. J Neurosci, 34(50), 16662-16670. doi: 10.1523/JNEUROSCI.2365-14.2014

van Kesteren, M. T., Rijpkema, M., Ruiter, D. J., Morris, R. G., \& Fernandez, G. (2014). Building on prior knowledge: schema-dependent encoding processes relate to academic performance. $J \operatorname{Cogn}$ Neurosci, 26(10), 2250-2261. doi: 10.1162/jocn_a_00630

van Kesteren, M. T. R., Beul, S. F., Takashima, A., Henson, R. N., Ruiter, D. J., \& Fernández, G. (2013). Differential roles for medial prefrontal and medial temporal cortices in schema-dependent encoding: from congruent to incongruent. Neuropsychologia, 51(12), 2352-2359.

van Kesteren, M. T. R., Fernandez, G., Norris, D. G., \& Hermans, E. J. (2010). Persistent schemadependent hippocampal-neocortical connectivity during memory encoding and postencoding rest in humans. Proc Natl Acad Sci U S A, 107(16).

van Kesteren, M. T. R., Rijpkema, M., Ruiter, D. J., \& Fernandez, G. (2010). Retrieval of associative information congruent with prior knowledge is related to increased medial prefrontal activity and connectivity. Journal of Neuroscience, 30(47), 15888-15894.

van Kesteren, M. T. R., Ruiter, D. J., Fernández, G., \& Henson, R. N. (2012). How schema and novelty augment memory formation. Trends in Neurosciences, 35(4), 211-219.

von Zerssen, G. C., Mecklinger, A., Opitz, B., \& von Cramon, D. Y. (2001). Conscious recollection and illusory recognition: an event-related fMRI study. The European Journal of Neuroscience, 13(11), 2148-2156.

Wheeler, M. E., \& Buckner, R. L. (2003). Functional dissociation among components of remembering: control, perceived oldness, and content. Journal of Neuroscience, 23(9), 3869-3880.

Wheeler, M. E., \& Buckner, R. L. (2004). Functional-anatomic correlates of remembering and knowing. Neuroimage, 21(4), 1337-1349.

Wheeler, M. E., Petersen, S. E., \& Buckner, R. L. (2000). Memory's echo: vivid remembering reactivates sensory-specific cortex. Proc Natl Acad Sci U S A, 97(20), 11125-11129.

Wise, R. J. S., \& Price, C. J. (2006). Functional imaging of language. In R. Cabeza \& A. Kingstone (Eds.), Handbook of functional neuroimaging of cognition (2nd ed) (pp. 191-228). Cambridge, MA: MIT Press.

Yonelinas, A. P. (2002). The nature of recollection and familiarity: A review of 30 years of research. Memory and Language, 46, 441-517.

Yonelinas, A. P., Hopfinger, J. B., Buonocore, M. H., Kroll, N. E., \& Baynes, K. (2001). Hippocampal, parahippocampal and occipital-temporal contributions to associative and item recognition memory: an fMRI study. Neuroreport, 12(2), 359-363.

Yonelinas, A. P., \& Jacoby, L. L. (1995). The relation between remembering and knowing as bases for recognition: Effects of size congruency. Journal of Memory and Language, 34(5), 622-643.

Yonelinas, A. P., Otten, L. J., Shaw, K. N., \& Rugg, M. D. (2005). Separating the brain regions involved in recollection and familiarity in recognition memory. Journal of Neuroscience, 25(11), 30023008 . 